\title{
Pragmatic Approach to Gravitational Radiation Reaction in Binary Black Holes
}

\author{
Carlos O. Lousto \\ Max-Planck-Institut für Gravitationsphysik, Albert-Einstein-Institut, Am Mühlenberg 1, D-14476 Golm, Germany, \\ and Instituto de Astronomía y Física del Espacio-CONICET, Buenos Aires, Argentina
}

(Received 8 October 1999)

\begin{abstract}
We study the relativistic orbit of binary black holes in systems with small mass ratio. The trajectory of the smaller object (another black hole or a neutron star), represented as a particle, is determined by the geodesic equation on the perturbed massive black hole spacetime. Here we study perturbations around a Schwarzschild black hole using Moncrief's gauge invariant formalism. We decompose the perturbations into $\ell$ multipoles to show that all $\ell$-metric coefficients are $C^{0}$ at the location of the particle. Summing over $\ell$, to reconstruct the full metric, gives a formally divergent result. We succeed in bringing this sum to a Riemann's $\zeta$-function regularization scheme and numerically compute the first-order geodesics.
\end{abstract}

PACS numbers: 04.30.-w, 04.25.Nx, 04.70.Bw

The computation of the gravitational radiation generated by binary black holes is of great theoretical and observational interest. On one hand these binary systems are among the best candidates to be detected by the new generation of gravitational wave detectors. On the other hand, the theoretically very interesting two body problem in general relativity remains an unsolved task for full numerical approaches. An appropriate astrophysical model to compute gravitational radiation coming from the capture of stars by massive black holes at the center of galaxies is provided by the perturbative approach applied to binary systems where one black hole is much more massive than the other. In the perturbative regime, linearized Einstein equations can be brought to two simple wave equations for the two polarizations of the gravitational field [1,2]. This problem has been recently revisited to include initial data into the formulation in order to be able to start numerical integrations from finite separations of the holes [3]. It also proved to be an excellent arena where to test the accuracy of the longitudinal-conformally flat ansatz to solve the initial value problem for binary black holes $[4,5]$. In order to answer questions such as what is the displacement of the innermost stable circular orbit (ISCO) and the rate of increase of the characteristic gravitational frequency due to inspiraling orbits, it is important to go beyond the leading approximation. Technically, one has to compute the geodesic trajectory of a particle in the perturbed black hole spacetime generated by the particle itself. Taking this effect into account will make otherwise particle's bounded orbits actually to inspiral towards the bigger black hole. This consistent description of the first-order perturbative approach opens the door to second-order perturbative studies [6] which allow one to compute gravitational radiation from binary black holes with much higher accuracy and for systems with not so small mass ratio. Such studies would teach us about new nonlinear physical effects as well as produce accurate templates to analyze the forthcoming data from ground (and space) based laser interferometric detectors.
Recent work on gravitational radiation reaction [7,8] prompted a renewed theoretical interest in this problem. The approach we will develop here privileges its direct computational implementability. Throughout this paper we will use results and techniques of Ref. [4]. There it was computed the gravitational radiation generated by the collision of two nonspinning black holes, one much less massive than the other, starting from rest at a finite distance. The extreme mass ratio allowed us to describe the problem of gravitational radiation as perturbations about the Schwarzschild metric. Hence, instead of working with all ten metric perturbations, seven even parity and three odd parity (that identically vanish for our axially symmetric problem), the relevant perturbative information is organized into (one in the even parity case, two in general) the Moncrief waveform [9]

$\psi_{\ell}(r, t)=\frac{r}{\lambda+1}\left[K^{\ell}+\frac{r-2 M}{\lambda r+3 M}\left\{H_{2}^{\ell}-r \partial K^{\ell} / \partial r\right\}\right]$,

where we have used Zerilli's [2] normalization for $\psi_{\ell}$ and notation for $\lambda \doteq(\ell+2)(\ell-1) / 2$.

One of the advantages of working with $\psi_{\ell}$ is its gauge invariance under first-order diffeomorphisms. This allows us to choose any convenient gauge, like the Regge-Wheeler gauge [1], to make computations. $\psi_{\ell}$ satisfies a single (in general two, with the odd parity case) wave equation

$$
-\frac{\partial^{2} \psi_{\ell}}{\partial t^{2}}+\frac{\partial^{2} \psi_{\ell}}{\partial r^{* 2}}-V_{\ell}(r) \psi_{\ell}=S_{\ell}(r, t),
$$

where $r^{*} \equiv r+2 M \ln (r / 2 M-1), V_{\ell}$ is the Zerilli potential, and $S_{\ell}(r, t)$ is the source term generated by the small hole, given in Ref. [3]. The smaller hole is described as a point particle of proper mass $m_{0}$, its stress energy tensor given by

$$
T^{\mu \nu}=m_{0} \frac{U^{\mu} U^{\nu}}{U^{0} r^{2}} \delta\left[r-r_{p}(t)\right] \delta^{2}[\Omega],
$$


where $U^{\mu}$ is the particle 4-velocity. The two dimensional delta function $\delta^{2}[\Omega]$ gives the angular location of the particle $\left(\theta_{p}, \phi_{p}\right)$. Since $T^{\mu \nu}$, and hence $S_{\ell}(r, t)$ are already proportional to $m_{0}$, to first perturbative order, the radial trajectory $r_{p}(t)$, follows from the geodesic equation in the background geometry (Schwarzschild's here). There are situations when one wants to know the trajectory of the small hole to the next order. In the computation of the gravitational radiation to second perturbative order one needs to know the source term to second order, hence the trajectory of the hole on the first-order metric (background plus first-order perturbations). Even within first-order perturbation theory one would also like to go further in order to compute secular effects like the particle's bounded orbit decay around a much bigger black hole.

For the sake of simplicity we will treat here the radial infall of a particle into a nonrotating hole. This problem contains many of the relevant features that occur for more general orbits. To first perturbative order, the trajectory of the particle is given by a geodesic on the first-order metric (Schwarzschild plus first-order perturbations). This is so because the only "forces" acting on the particle are gravitational. The time component of the particle's four-momentum, $\quad P_{t}=m_{0} g_{t t}(d t / d \tau) \doteq-m_{0} E$, is no longer a conserved quantity along the trajectory. We then have to deal with the radial and time (here the only nontrivial ones) components of the geodesic equation. We combine these two equations into a single equation of motion for $r(t)$ by eliminating the dependence on the geodesic parameter. Linearization of the resulting equation and subtraction of the zeroth-order geodesic gives the deviation of the trajectory $\Delta r_{p}(t)$ from the zeroth-order one $\dot{r}_{p}(t)=\partial_{t} z_{p}=-\left(1-2 M / z_{p}\right) \sqrt{\frac{2 M / z_{p}-2 M / z_{0}}{1-2 M / z_{0}}}$, directly in terms of Schwarzschild coordinates, ready for further applications

$$
\Delta \ddot{r}_{p}=A \Delta r_{p}+B \Delta \dot{r}_{p}+C,
$$

where

$$
K^{\ell}=\frac{6 M^{2}+3 M \lambda r+\lambda(\lambda+1) r^{2}}{r^{2}(\lambda r+3 M)} \psi_{\ell}+\left(1-\frac{2 M}{r}\right) \partial_{r} \psi_{\ell}-\frac{\kappa U^{0}(r-2 M)^{2}}{(\lambda+1)(\lambda r+3 M) r} \delta\left[r-r_{p}\right]
$$

and

$$
\begin{aligned}
H_{2}^{\ell}= & -\frac{9 M^{3}+9 \lambda M^{2} r+3 \lambda^{2} M r^{2}+\lambda^{2}(\lambda+1) r^{3}}{r^{2}(\lambda r+3 M)^{2}} \psi_{\ell}+\frac{3 M^{2}-\lambda M r+\lambda r^{2}}{r(\lambda r+3 M)} \partial_{r} \psi_{\ell}+(r-2 M) \partial_{r}^{2} \psi_{\ell} \\
& +\frac{\kappa U^{0}(1-2 M / r)\left[\lambda^{2} r^{2}+2 \lambda M r-3 M r+3 M^{2}\right]}{(\lambda+1)(\lambda r+3 M)^{2}} \delta\left[r-r_{p}\right]-\frac{\kappa U^{0}(r-2 M)^{2}}{(\lambda+1)(\lambda r+3 M)} \delta^{\prime}\left[r-r_{p}\right] .
\end{aligned}
$$

Integration over $r$ of the Hamiltonian constraint tells us that the leading behavior is given by $\partial_{r} \psi_{\ell} \sim \kappa U^{0}(r-$ $2 M) \delta\left[r-r_{p}\right] /(\lambda+1) /(\lambda r+3 M)$. This can be used to prove that the metric coefficients at the location of the par- ticle are actually $C^{0}$, by taking up to second derivatives and using the equation above to cancel derivatives of the Dirac's delta. The same $C^{0}$ behavior at $r_{p}$ can be proved for $H_{1}^{\ell}$. We now consider the $t r$ and $t \theta$ (or $t \varphi$ ) components 
of the Einstein equations that give us the momentum constraint. In the Regge-Wheeler gauge they are given by Zerilli's [2] Eqs. (C7b) and (C7d). We combine them to eliminate the dependence on $H_{1}^{\ell}$, and obtain after integration over $r$ that $\partial_{r}\left(\partial_{t} \psi_{\ell}\right) \sim-\kappa U^{0} \dot{r}_{p}(r-2 M) \partial_{r} \delta[r-$ $\left.r_{p}\right] /(\lambda+1) /(\lambda r+3 M)$.

From Zerilli's [2] Eq. (C7b) and the expressions for $\partial_{t} K^{\ell}$ and $\partial_{t} H_{2}$ in terms of $\partial_{t} \psi_{\ell}$, we find the last metric coefficient in the Regge-Wheeler gauge

$$
\begin{aligned}
H_{1}^{\ell}= & r \partial_{r}\left(\partial_{t} \psi_{\ell}\right)+\frac{\lambda r^{2}-3 M \lambda r-3 M^{2}}{(r-2 M)(\lambda r+3 M)} \partial_{t} \psi_{\ell} \\
& -\frac{\kappa U^{0} \dot{r}_{p}(\lambda r+M)}{(\lambda+1)(\lambda r+3 M)} \delta\left[r-r_{p}\right] \\
& +\frac{\kappa U^{0} \dot{r}_{p} r(r-2 M)}{(\lambda+1)(\lambda r+3 M)} \delta^{\prime}\left[r-r_{p}\right] .
\end{aligned}
$$

This equation together with $H_{0}^{\ell}=H_{2}^{\ell}$ (valid for head-on collisions), (6), and (7) give us all metric perturbations on the chosen hypersurface in terms only of $\psi_{\ell}$ and $\partial_{t} \psi_{\ell}$ (and the source). We recall here that since our case has axial symmetry only even waves are generated.

In Fig. 1 we plot the results of computing by means of Eq. (7) the metric coefficient $\tilde{H}_{2} \doteq(1-$ $\left.2 M / r_{p}\right) \sqrt{2 \ell+1} H_{2}^{\ell}$ along the trajectory of the particle $r_{p}(t)$. It clearly shows that it is finite at each point of the trajectory. The other notable feature is that for large $\ell$ the curves quickly accumulate over the $\ell \rightarrow \infty$ asymptotic curve. Since one has then to sum over all $\ell$ contributions, this sum clearly diverges. One way of regularizing this sum is to subtract to each mode precisely the $\ell \rightarrow \infty$ contribution, and then verify the convergence

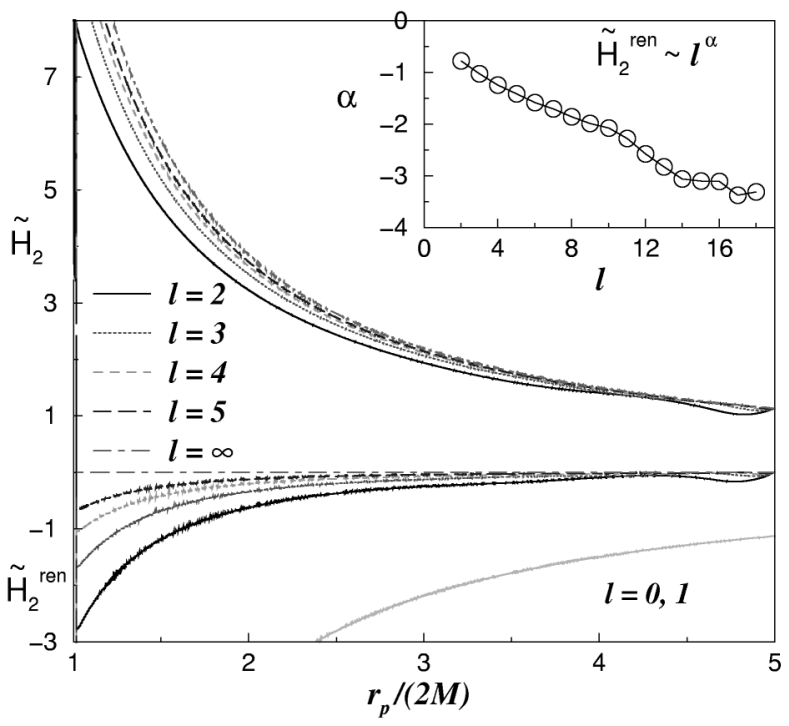

FIG. 1. The upper part of the plot shows the metric coefficient $\tilde{H}_{2}^{\ell}$ along the trajectory of the particle in units of $m_{0}$. The lower part of the plot represents the renormalized metric $\tilde{H}_{2}^{\ell \text { ren }}=\tilde{H}_{2}^{\ell}-\tilde{H}_{2}^{\infty}$. The inset figure gives the leading decay power, $\alpha$, of $H_{2}^{\ell \text { ren }}$ at a fiducial $r_{p}$. of the remanent series. The result of such regularization is shown in the lower half of Fig. 1. The same qualitative results are found for the other metric coefficients $H_{1}^{\ell}\left(r_{p}\right)$, $K^{\ell}\left(r_{p}\right)$, and $H_{0}^{\ell}\left(r_{p}\right)=H_{2}^{\ell}\left(r_{p}\right)$. Nonradiative multipoles $\ell=0,1$ can be found analytically and represent the mass and linear momentum contributions of the particle to the system [2]. The regularization just described is ambiguous up to a finite piece. To give a definite prescription we note that it can be brought into the generalized Riemann's $\zeta$ function [10] procedure as follows. The numerical behavior of all metric coefficients shows that they can be decomposed into two pieces: One that generates the finite behavior for $\ell \rightarrow \infty$ and the other strongly decaying for large $\ell$ (labeled with a ren below). Thus, for instance, for the perturbed metric component $g_{r r}$ we can write

$$
\begin{aligned}
H_{2}(t, r, \theta, \phi)= & \sum_{\ell m} H_{2}^{\ell m}(t, r) Y_{\ell m}(\theta, \phi) \\
= & \sum_{\ell=0}^{\infty}\left\{(2 \ell+3 / 2-\beta)^{-\beta} H_{2}^{\infty}+H_{2}^{\ell \text { ren }}\right\} \\
& \times \sqrt{\frac{2 \ell+1}{4 \pi}} P_{\ell}(\theta),
\end{aligned}
$$

where in the second equality we made use of the axial symmetry of the problem and we have chosen the $\beta$ parametrization motivated by the $D$-dimensional extension of the conformally flat initial value problem, where $\beta=9 / 2-D$. When we evaluate this metric coefficient at the location of the particle we find

$$
\begin{aligned}
H_{2}\left[t, r_{p}(t), \theta_{p}=\right. & 0] \\
= & 2^{-\beta+1 / 2} \frac{H_{2}^{\infty}}{\sqrt{4 \pi}} \zeta(\beta-1 / 2,1 / 2) \\
& +\sum_{\ell=0}^{\infty} \sqrt{\frac{2 \ell+1}{4 \pi}} H_{2}^{\ell \text { ren }} .
\end{aligned}
$$

where the Riemann's $\zeta$ function is $\zeta(a, b)=\sum_{\ell=0}^{\infty} \times$ $(\ell+b)^{-a}$. Numerically, we observe that $\beta=1 / 2$ in order to lead to the finite $\ell \rightarrow \infty$ behavior. Since the analytically continued $\zeta$ function gives $\zeta(0,1 / 2)=0$ we must subtract to each multipole just the $\ell \rightarrow \infty$ piece. The renormalized metric coefficients that enter into the head-on geodesic equation $\left(H_{0}^{\ell \text { ren }}=H_{2}^{\ell \text { ren }}, H_{1}^{\ell \text { ren }}\right)$ vanish for large $\ell$ as $\sim \ell^{-3}$, as we numerically roughly estimated (cf. Fig. 1). This implies that the regularized connection coefficients scale as $\sim \ell^{-2}$ for large $\ell$ and that its sum, to compute $C$, up to a finite maximum $L$ (as is done in practice) scale as $\sim L^{-1}$. This can be used to estimate the error produced by a truncation of the series.

From the metric we build up the connection coefficients that enter into the geodesic equation. The piece of the relative acceleration that is summed over $\ell$, denoted by $C$ in Eq. (5), needs to be regularized in the same way metric coefficients do. This piece summed over the 

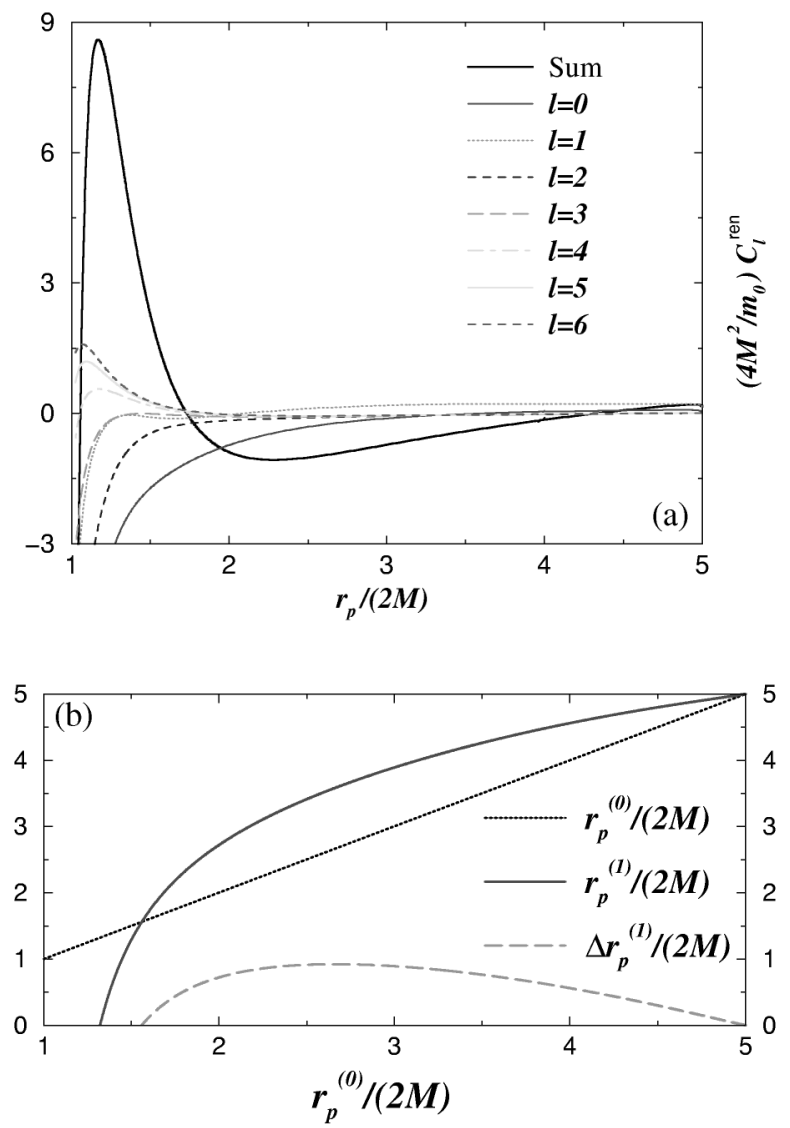

FIG. 2. The renormalized radiative piece of the reaction on $\ddot{r}_{p}$. We show the regularized sum over $\ell \leq 10$ multipole contributions to $C$, as defined in Eq. (5). Below, the first-order trajectory $r_{p}^{(1)}$, for $m_{0}=0.1 M$, is compared to the zeroth order one, $r_{p}^{(0)}$.

$\ell \leq 10$ radiative multipoles is plotted in Fig. $2(\ell=0$ and $\ell=1$ multipoles have been solved analytically using the additional gauge freedom to set $H_{2}=K$ and, in the $\ell=0$ case, also $H_{1}=0$ ). We find the radiation reaction effects can be qualitatively described in two regimes. First, the renormalized acceleration is very small in the early stages of the trajectory. This is expected for a particle starting at rest, since the gravitational radiation is dominated by the bremsstrahlung mechanism. Then, radiation reaction effects become more important as the particle approaches the maximum of the Zerilli's potential (around $\left.r_{\max } \approx 3.1 M\right)$. They tend to decelerate the particle with respect to the zeroth order (Schwarzschild) geodesics. This is what one would qualitatively expect a priori since the system is losing energy and momentum in the form of gravitational radiation. Another important feature is that the radiation reaction effect is low $\ell$-dominated (only after renormalization), but still the sum over large $\ell$ gives an important contribution, and one has to consider higher multipoles than one usually takes into account when computing, for instance, the total energy radiated reaching infinity.
A related approach to the one presented here has been developed independently [11] and applied to scalar radiation. It considers the large $\ell$ dependence of the tail term of the reaction force [8], and regularizes the sum over $\ell$ modes by subtracting the nonconvergent terms plus a finite part to be determined. Applied to the equivalent case we treated here the finite part to be subtracted vanishes and both procedures coincide. Besides, this method was implemented to study static scalar and electric fields in the Schwarzschild background [12], leading to the correct known expressions of the self-force.

Here we reported on a first important step to improve our ability to compute gravitational radiation from binary black holes. The next step is to apply the $\zeta$-function regularization method to general bounded orbits in the Schwarzschild background and compare its results with the energy and momentum balance estimates. Besides, our renormalization procedure is explicitly gauge invariant since we use Moncrief's waveform. Another key problem to attack is the orbiting particle in a rotating black hole background. Perturbations of the Kerr metric are described by the Teukolsky equation and can be decomposed into multipoles (via spin-weighted spheroidal harmonics) in the frequency domain, i.e., after a Fourier decomposition of the time dependence. This property would still allow us to apply our regularization scheme and compute the decay of bounded orbits consistently to first order. Besides, it would open the possibility to study second-order perturbations [6] and obtain a remarkable improvement in our ability to compute gravitational radiation from binary systems with not so small mass ratio. This will not only be of use for LISA's detection of gravitational radiation from black holes in the center of nearby galaxies, but also relevant for ground based interferometers, sensible to frequencies corresponding to black hole/ neutron star binaries.

[1] T. Regge and J. A. Wheeler, Phys. Rev. 108, 1063 (1957).

[2] F. J. Zerilli, Phys. Rev. Lett. 24, 737 (1970).

[3] C. O. Lousto and R. Price, Phys. Rev. D 55, 2124 (1997).

[4] C. O. Lousto and R. Price, Phys. Rev. D 56, 6439 (1997).

[5] C. O. Lousto and R. Price, Phys. Rev. D 57, 1073 (1998).

[6] M. Campanelli and C. O. Lousto, Phys. Rev. D 59, 124022 (1999).

[7] Y. Mino, M. Sasaki, and T. Tanaka, Phys. Rev. D 55, 3457 (1997).

[8] T. C. Quinn and R. Wald, Phys. Rev. D 56, 3381 (1997).

[9] V. Moncrief, Ann. Phys. (N.Y.) 88, 323 (1974).

[10] N.D. Birrell and P.C.W. Davies, Quantum Fields in Curved Space (Cambridge University Press, Cambridge, England, 1982).

[11] L. Barack and A. Ori, Phys. Rev. D 61, 061502 (2000).

[12] L. M. Burko, Classical Quantum Gravity 17, 227 (2000). 